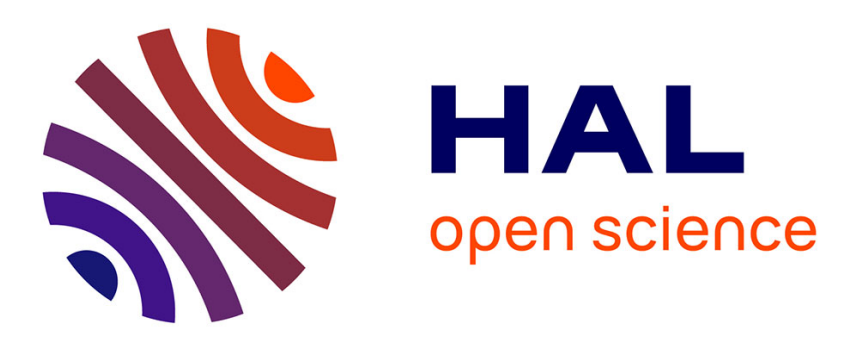

\title{
Etude de la solidification directionnelle en creuset du silicium en vue d'un usage solaire
}

\author{
G. Revel, J.-L. Pastol, D. Hania, Nguyen Dinh Huynh
}

\section{To cite this version:}

G. Revel, J.-L. Pastol, D. Hania, Nguyen Dinh Huynh. Etude de la solidification directionnelle en creuset du silicium en vue d'un usage solaire. Revue de Physique Appliquée, 1987, 22 (7), pp.519-528. 10.1051/rphysap:01987002207051900 . jpa-00245571

HAL Id: jpa-00245571

https://hal.science/jpa-00245571

Submitted on 1 Jan 1987

HAL is a multi-disciplinary open access archive for the deposit and dissemination of scientific research documents, whether they are published or not. The documents may come from teaching and research institutions in France or abroad, or from public or private research centers.
L'archive ouverte pluridisciplinaire HAL, est destinée au dépôt et à la diffusion de documents scientifiques de niveau recherche, publiés ou non, émanant des établissements d'enseignement et de recherche français ou étrangers, des laboratoires publics ou privés. 
Classification

Physics Abstracts

$82.40-91.10-91.18$

\title{
Etude de la solidification directionnelle en creuset du silicium en vue d'un usage solaire
}

\author{
G. Revel (*), J.-L. Pastol, D. Hania et Nguyen Dinh Huynh (**) \\ C.N.R.S., Centre d'Etudes de Chimie Métallurgique, 15, rue Georges Urbain, 94407 Vitry-sur-Seine Cedex, \\ France \\ (*) C.N.R.S., Laboratoire d'Analyse par Activation «Pierre Süe», C.E.N. Saclay, 91191 Gif-sur- \\ Yvette Cedex, France \\ (**) C.N.R. Vietnam, Centre d'Etudes pour l'Utilisation de l'Energie Solaire, 1 Mac Dinh Chi, Hochiminh- \\ ville, Vietnam
}

(Reçu le 20 octobre 1986, accepté le 4 mars 1987)

\begin{abstract}
Résumé. - La méthode de croissance développée permet de préparer des lingots de silicium mono et polycristallins. La solidification est réalisée de bas en haut à partir d'un échangeur de chaleur. Divers matériaux ont été testés pour la réalisation du creuset. Des échantillons de qualités électronique (Si-EG) et métallurgique améliorée (Si-UMG) sont traités. Cette méthode permet également de préparer des lingots de Si-EG enrichis volontairement en une impureté sélectionnée. Les lingots obtenus sont caractérisés par macro et micrographie, analyses par activation et mesures de propriétés électriques. L'influence des conditions d'échange thermique sur la forme de l'interface et ses conséquences sur la structure des lingots est abordée. Le comportement des impuretés au cours de la solidification est étudié. La possibilité de réaliser des cellules solaires à partir de Si-UMG est discutée.
\end{abstract}

\begin{abstract}
The growth method developed allows to prepare single and polycrystalline silicon ingots. Solidification takes place from the bottom to the top from a heat exchanger. Some crucible materials have been tested. Electronic grade (EG-Si) and upgraded metallurgical grade (UMG-Si) silicon samples are treated. This method allows too to prepare EG-Si ingots deliberately enriched with a selected impurity. The obtained ingots are studied by macro and micrography, activation analyses and electrical properties measurements. The influence of thermal exchange conditions on the interface shape and the consequences on the structure of the ingots is considered. The behaviour of the impurities during the solidification is studied. The possibility to make solar cells from UMG-Si ingots is discussed.
\end{abstract}

\section{Introduction.}

Depuis une dizaine d'années, des efforts considérables ont été faits pour abaisser le coût des panneaux solaires réalisés à partir de silicium massif. Un facteur sept à dix a été gagné sur les prix de revient. Par contre, la part du silicium de départ dans le coût total du panneau n'a cessé de croître. De plus, il existe actuellement une pénurie des sources d'approvisionnement en silicium pour un usage solaire. Aussi de nombreux travaux sont faits partout dans le monde pour obtenir un silicium de qualité dite « solaire » qui serait un peu moins parfait et beaucoup moins coûteux que le silicium de qualité «électronique» utilisé précédemment pour cet usage.

REVUE DE PHYSIQUe APPLIQUÉE. - T. 22, N` 7, JUILLET 1987
Une première étape a été franchie en abandonnant progressivement le silicium monocristallin tiré selon la méthode de Czochralski au profit de silicium polycristallin refondu et solidifié dans un creuset. Une économie importante a ainsi été réalisée sur les coûts d'investissements et de fonctionnement pour la recristallisation du silicium, mais des progrès décisifs restent à faire sur le coût du silicium utilisé comme charge à recristalliser.

Plusieurs voies sont explorées : amélioration de la voie chimique, récupération de silicium déclassé de l'électronique, amélioration du silicium de qualité métallurgique.

Les différents procédés actuels d'élaboration de lingots polycristallins : SILSO, POLYX, HEM... 
engendrent une modification importante de la teneur et de la répartition des impuretés présentes dans la charge de départ. La fusion entraîne un risque de pollution non négligeable par le creuset ou par ses impuretés. La solidification peut permettre l'élimination des impuretés par ségrégation dans les parties solidifiées en dernier. Enfin, certaines impuretés peuvent aussi s'éliminer par évaporation.

Les performances photoélectriques obtenues dépendront non seulement de la pureté globale de la charge, mais également de la nature, de la répartition des impuretés et de la structure cristalline des lingots obtenus.

\section{Etude de la méthode de croissance.}

Nous avons développé une méthode de croissance directionnelle utilisant un échangeur de chaleur. Le principe de cette méthode, connue sous le nom de HEM (marque déposée de Crystal Systems, U.S.A.) est simple et a fait l'objet de nombreuses publications $[1,2]$. Il consiste à solidifier directionnellement un liquide à partir du fond du creuset en extrayant la chaleur au moyen d'un échangeur de chaleur. Ce procédé a été, à l'origine, proposé pour préparer des monocristaux de grandes dimensions [3], mais il permet également de préparer des polycristaux à gros grains.

Nous avons choisi cette méthode car elle est proche des autres méthodes de solidification direc- tionnelles en creuset de type Bridgman (par exemple POLYX [4]) ; de plus, elle permet d'obtenir, dans des conditions similaires, des monocristaux et des polycristaux et l'utilisation d'un échangeur de chaleur apporte un meilleur contrôle de la solidification.

Nous avons développé cette méthode au C.E.C.M. de Vitry dans le cadre de trois contrats COMES et AFME [5-7]. L'installation que nous avons conçue et réalisée pour mettre en œuvre ce procédé a déjà été décrite [8]. La charge de silicium est placée dans un creuset (diamètre $100 \mathrm{~mm}$, hauteur $160 \mathrm{~mm}$ ). La fusion de la charge est totale. Sa solidification est réalisée de bas en haut, soit à partir d'un germe placé sur l'échangeur de chaleur pour les essais visant à obtenir un monocristal, soit directement à partir du fond du creuset pour des essais visant à obtenir un polycristal à structure basaltique. La vitesse de déplacement du front de solidification est de l'ordre de $1 \mathrm{~cm} / \mathrm{h}$.

Plus de trente lingots ont été ainsi préparés, soit à partir de silicium de qualité électronique : «Si-EG » (9 N), soit à partir de silicium de qualité métallurgique améliorée par lavage acide : «Si-UMG» (3$4 \mathrm{~N}$ ). Certains de ces lingots ont été préparés par solidification à partir d'un germe monocristallin, d'autres par solidification à partir du fond du creuset. Des essais ont été effectués à partir de Si-UMG en poudre. Plusieurs lingots de Si-EG dopés volontairement en une impureté sélectionnée $(\mathrm{Al}, \mathrm{B}, \mathrm{C}, \mathrm{Cu})$ ont été réalisés pour étudier plus particulièrement le

Tableau I. - Résultats obtenus avec les creusets testés.

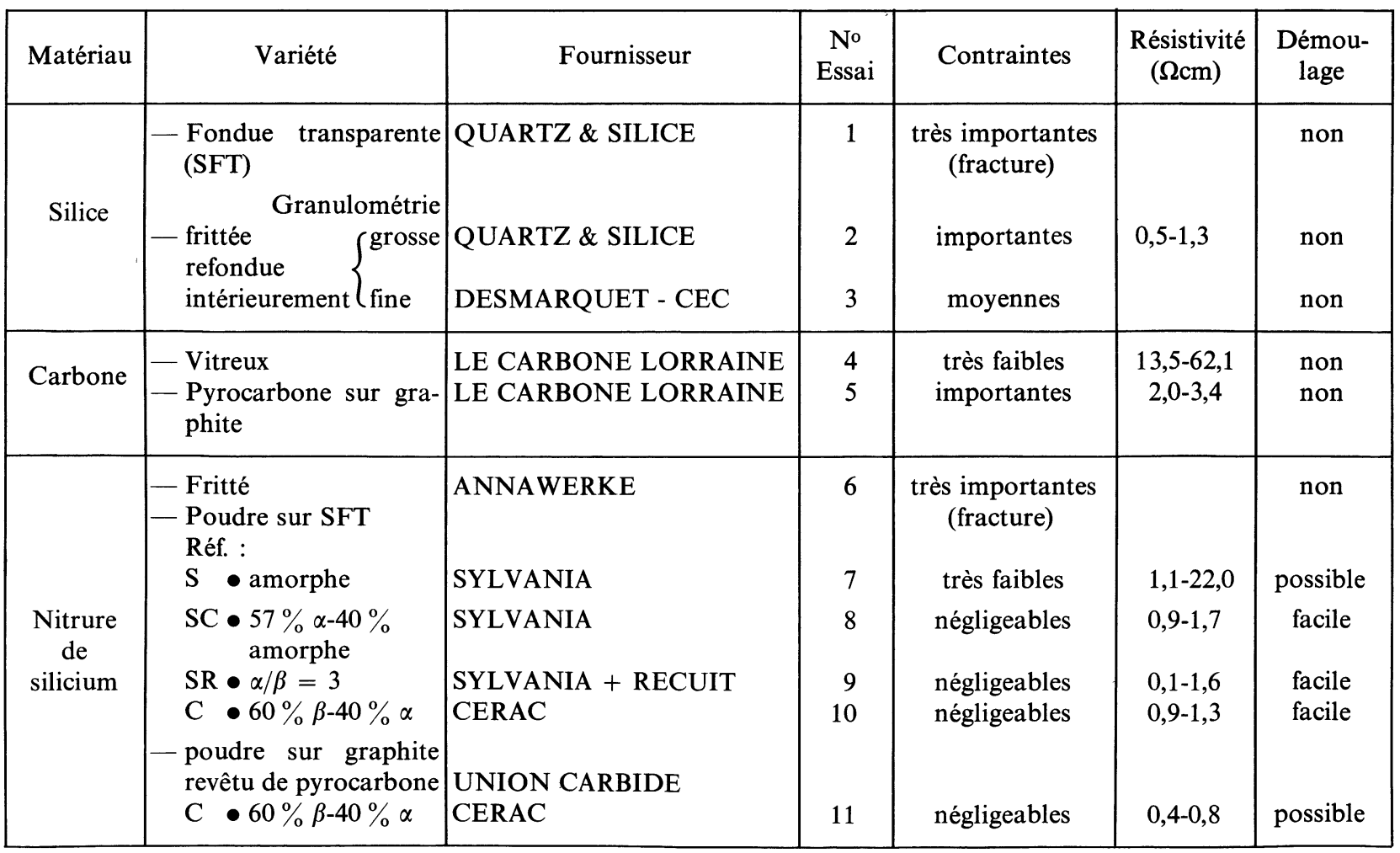


comportement de cette impureté au cours de la solidification et son influence sur les propriétés électriques du silicium.

\section{Choix du creuset.}

De nombreux matériaux à base de silice, de carbone et de nitrure de silicium ont été testés (Tableau I).

Notre choix s'est porté sur un creuset en silice fondue transparente revêtue intérieurement de nitrure de silicium [9]. Les pollutions introduites au cours de la fusion sont tolérables et les contraintes éventuellement introduites au cours du refroidissement n'engendrent pas de fissure dans les lingots. De plus, l'adhérence au creuset étant nulle et les coefficients de dilatation du silicium et de la silice différents, les lingots sont démoulés très facilement à température ambiante.

Cette solution a été adoptée par plusieurs Laboratoires et Sociétés au Japon [10], aux U.S.A. [11], en Italie [12]...

Pour un usage industriel, des efforts seraient encore à faire sur la qualité des revêtements nitrure (pureté, état cristallin). Les nitrures les plus purs sont vendus à l'état amorphe, mais c'est l'état cristallisé qui convient le mieux pour éviter les inclusions de nitrure dans les lingots.

Par ailleurs, les creusets en silice ne sont pas réutilisables car ils sont dévitrifiés à chaque traitement. Le graphite serait économiquement préférable, mais nos essais ont montré qu'il réagissait à chaud avec le nitrure. L'interposition d'une barrière de $\mathrm{SiC}$ entre le graphite et le nitrure pourrait être une solution [13]. Les dépôts de $\mathrm{SiC}$ que nous avons pu obtenir par CVD n'étaient pas assez adhérents aux creusets pour donner des résultats concluants.

\section{Caractérisation des lingots obtenus.}

Après solidification totale et refroidissement lent, les lingots sont démoulés à la température ambiante par simple retournement du creuset (Fig. 1) et découpés pour étude suivant le schéma de la figure 2 .

L'étude de la structure cristalline est faite par macroscopie et microscopie sur la plaque verticale VA. Cette plaque est aussi utilisée pour réaliser une double cartographie, par mesure de la résistivité électrique et par analyse par activation neutronique. Les prélèvements 1,2 et 3 sont parfois utilisés pour des analyses complémentaires aux photons gamma et aux particules chargées. Les observations en macroscopie et microscopie optique sont faites au C.E.C.M. de Vitry. La figure 3 rassemble les résultats obtenus sur les tranches verticales de quatre lingots préparés à partir de silicium de qualités électronique et métallurgique améliorée, solidifiés avec ou sans germe. Des observations complémentaires en microscopie électronique sont faites par J.-L. Maurice et J.-Y. Laval (E.R.A. 912). Enfin, pour

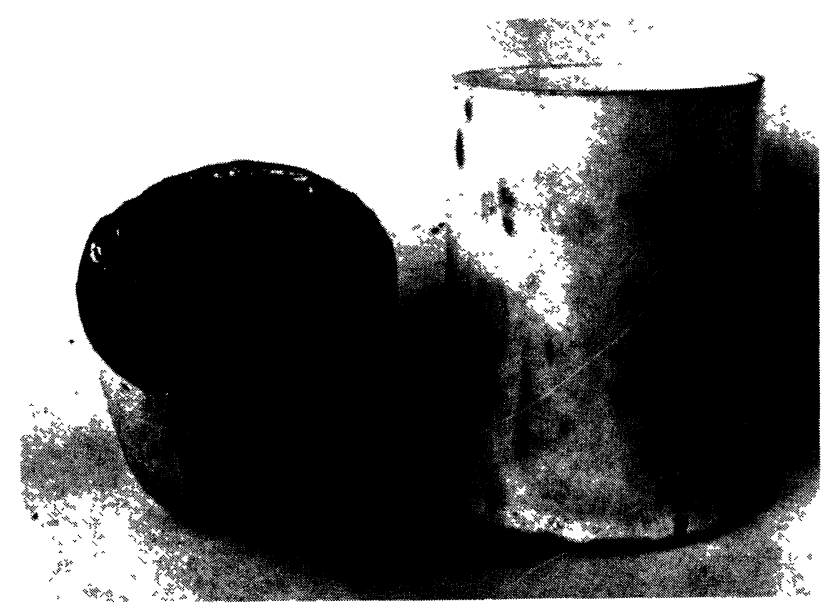

Fig. 1. - Lingot de silicium (diamètre $100 \mathrm{~mm}$ ) et creuset après démoulage.

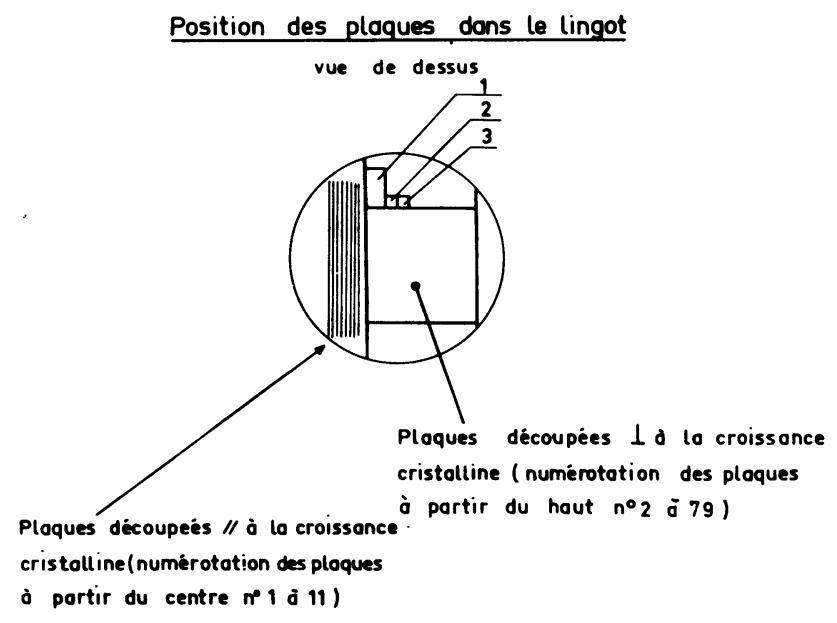

Fig. 2. - Schéma de découpe des lingots.

tenter d'identifier les précipités décelés dans les lingots préparés à partir de Si-UMG, quelques échantillons ont été examinés à la sonde électronique par Mme Rommeluère du Laboratoire CNRS de Meudon-Bellevue. Les analyses par activation neutronique sont réalisées au Laboratoire Pierre Süe du C.E.N. Saclay suivant une méthode spécialement mise au point pour cette étude et déjà décrite $[14,15]$. Cette méthode permet de doser une quarantaine d'impuretés, métalliques pour la plupart. Les limites de détection obtenues sur les échantillons étudiés vont de $10^{9}$ à $10^{15}$ at. $\mathrm{cm}^{-3}$ suivant les éléments et les échantillons. Pour les éléments légers, quelques dosages complémentaires ont été faits par SIMS par B. Blanchard du C.E.N. Grenoble (bore, aluminium), par activation dans les photons gamma par J.-C. Rouchaud du C.E.C.M. de Vitry (carbone, azote) et par activation dans les particules chargées par C. Valladon du Laboratoire du Cyclotron d'Orléans (bore, carbone, oxygène).

Les résistivités électriques sont mesurées par la 


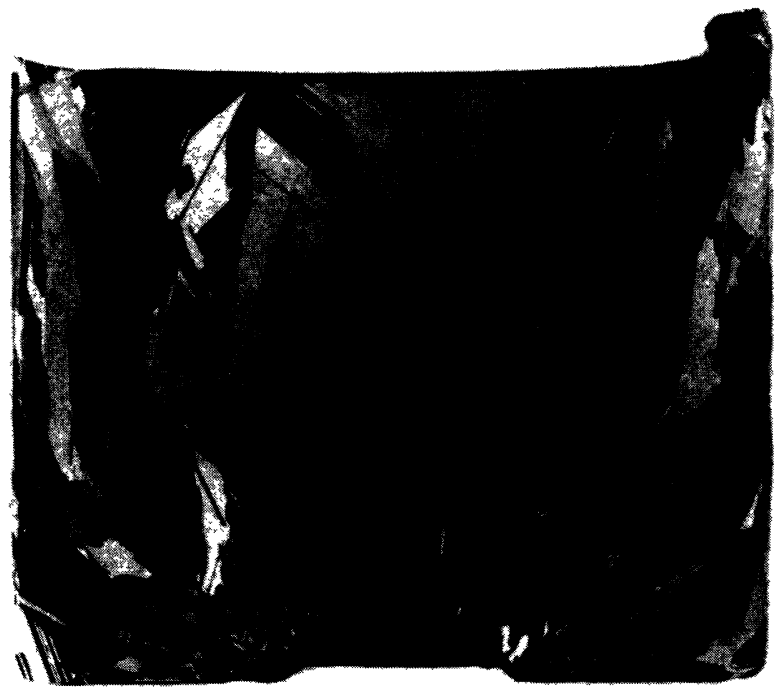

Lingot W/R5

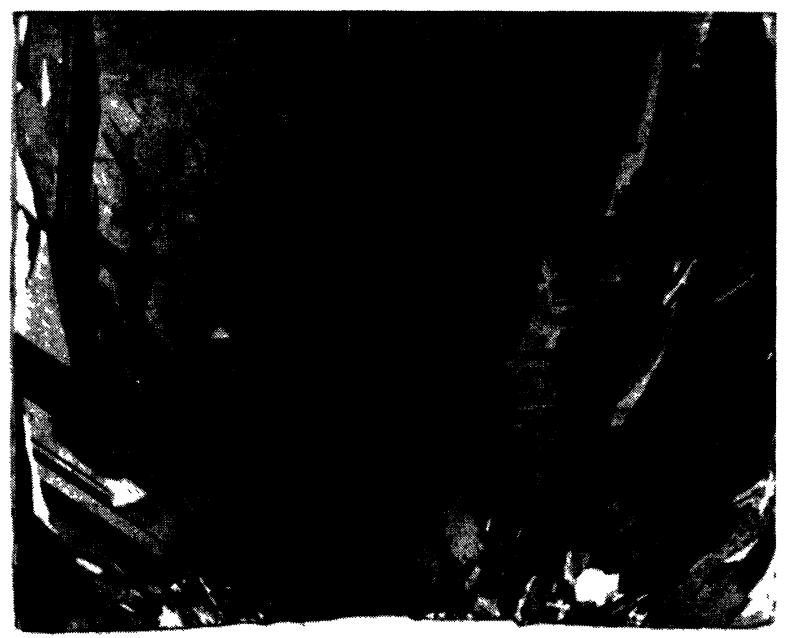

Lingot P4/R18

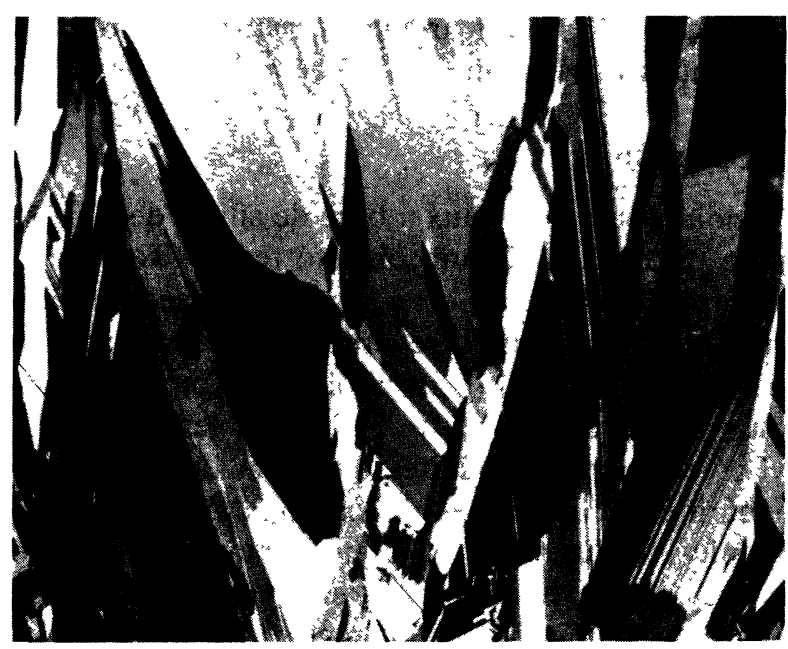

Lingot W/R17

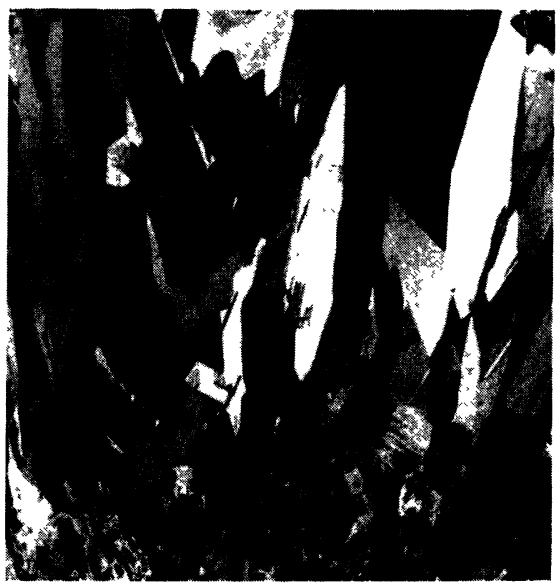

Lingot P2/R15

Fig. 3. - Coupe parallèle à l'axe des lingots : W/R5 : Si-EG avec germe ; W/R17 : Si-EG sans germe ; P4/R18: SiUMG avec germe ; P2/R15 : Si-UMG sans germe.

méthode des quatre pointes alignées, au Laboratoire de Physique du Solide de Meudon-Bellevue. La figure 4 rassemble les résultats obtenus sur les quatre plaques précédentes.

Les plaques verticales $\mathrm{V}$ et horizontales $\mathrm{H}$ sont utilisées pour réaliser des cellules selon la technique développée par la Société Photowatt à Caen $[16,17]$. La figure 5 rassemble les caractéristiques I(V) des quatre lingots déjà cités. A partir de Si-EG, les performances obtenues sont correctes et très voisines entre mono et polycristaux. A partir de SiUMG, les performances sont mauvaises [8].

Enfin, des dépôts en épitaxie ont été réalisés à la Faculté Catholique de Louvain, dans le Laboratoire ESAT de MM. les Professeurs Van Overstraeten et Mertens suivant une technique qu'ils ont développée $[18,19]$.
5. Forme de l'interface solide-liquide au cours de la solidification.

Cette forme peut être concave, plane ou convexe (Fig. 6). Elle dépend de la configuration thermique du dispositif de croissance, mais elle est difficile à observer directement au cours de la croissance. Cependant, il est indispensable de connaître et de contrôler cette forme car d'elle dépend la structure cristalline des lingots obtenus et donc les performances des photopiles que l'on pourra préparer à partir de ces lingots.

Nous avons soigneusement contrôlé les conditions de croissance de nos lingots et mesuré par palpage mécanique la position de l'interface tous les quarts d'heure au cours de la croissance. Les observations macroscopiques, ajoutées aux résultats des analyses et des mesures de résistivité, nous permettent d'asso- 


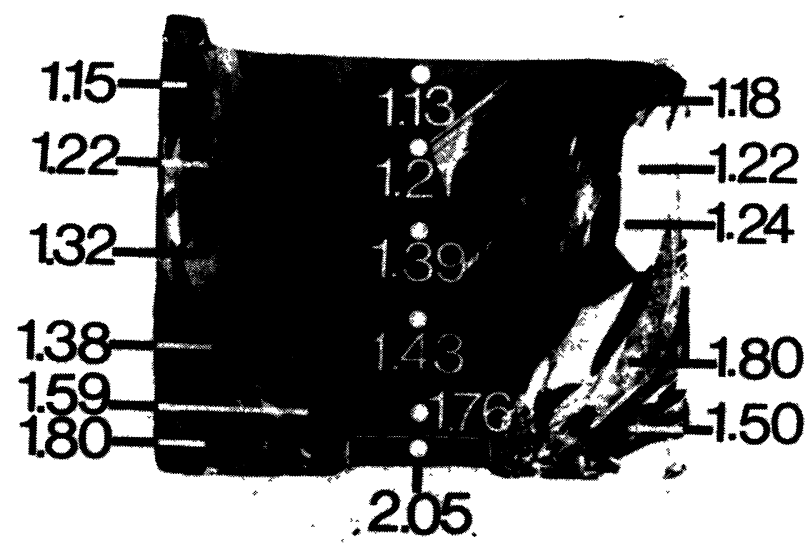

Lingot W/R5

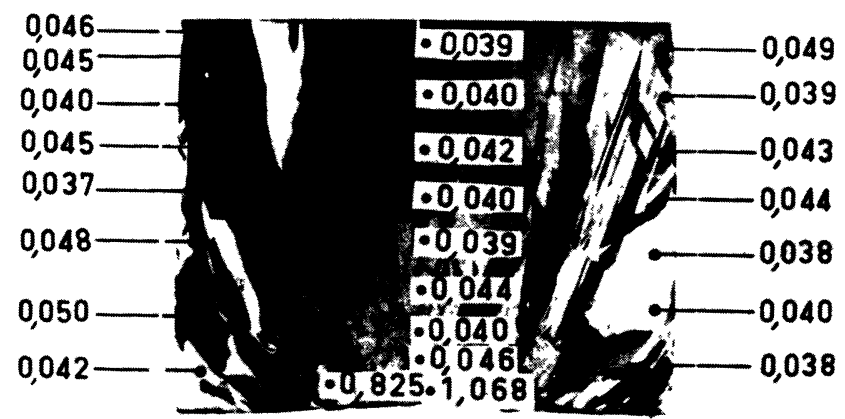

Lingot P4/R18

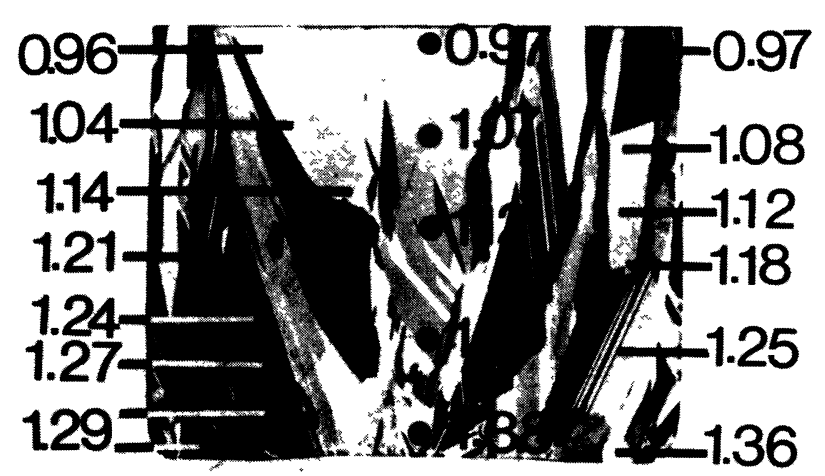

Lingot W/R17

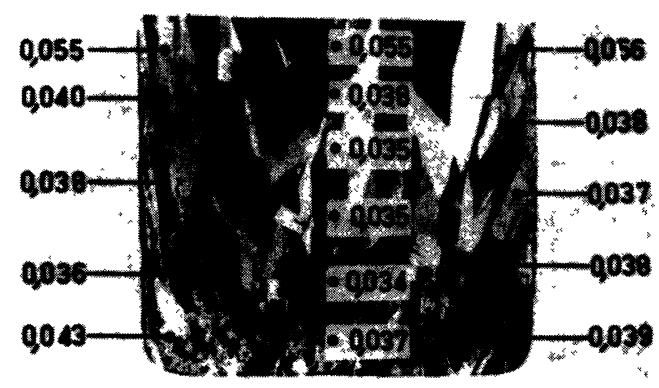

Lingot P2/R15

Fig. 4. - Résistivités en $\mathbf{\Omega} . \mathrm{cm}$ mesurées sur les plaques parallèles à l'axe des lingots : W/R5: Si-EG avec germe ; W/R17 : Si-EG sans germe ; P4/R18 : Si-UMG avec germe ; P2/R15 : Si-UMG sans germe.

cier la forme de l'interface aux conditions de traitement.

L'interface concave est à éviter car elle se traduit par des germinations parasites à partir des bords du creuset. Elle se produit lorsqu'une partie de la chaleur est extraite par les parois latérales (exemple procédé SILSO).

L'interface plane est très favorable pour obtenir des polycristaux à gros grains avec une structure basaltique ; elle est obtenue en extrayant la chaleur de façon uniforme par le fond du creuset (exemple procédé POLYX).

L'interface concave est nécessaire, au moins au début de la croissance, pour obtenir un monocristal à partir d'un germe (procédé HEM). Nous avons montré que le rayon de courbure de cette interface dépendait de la conductibilité thermique des parois du creuset ; lorsque celle-ci est faible, l'interface a tendance à devenir plane et la partie monocristalline diminue de volume pour ne plus occuper que la partie à l'aplomb du germe (Figs. 7 et 8 ). Il est à noter que lorsque la chaleur est extraite uniquement par le fond du creuset, à travers le silicium solidifié, la vitesse de déplacement de l'interface est forcément limitée.

Si l'on assimile l'échangeur de chaleur à un point au centre du fond du creuset l'interface solideliquide aura une forme hémisphérique. Dans ce cas, en régime permanent, on montre que pour une vitesse de croissance constante, le débit de l'hélium doit augmenter comme le carré de la hauteur solidifiée [5].

Un calcul développé dans le cas d'une interface plane montre que l'inertie thermique est multipliée par un facteur 100 lorsque la hauteur solidifiée passe de quelques centimètres à quelques dizaines de centimètres [20].

Dans la pratique, ceci signifie que l'augmentation de la taille des lingots doit être réalisée par l'augmen tation de leur surface et non par celle de leur hauteur; les productions industrielles ou semiindustrielles récentes concernent des lingots de $23 \times 23 \times\left(8\right.$ à 10) $\mathrm{cm}^{3}$ [21] et de $40 \times 40 \times$ (10 à 15) $\mathrm{cm}^{3}$ [22]. 

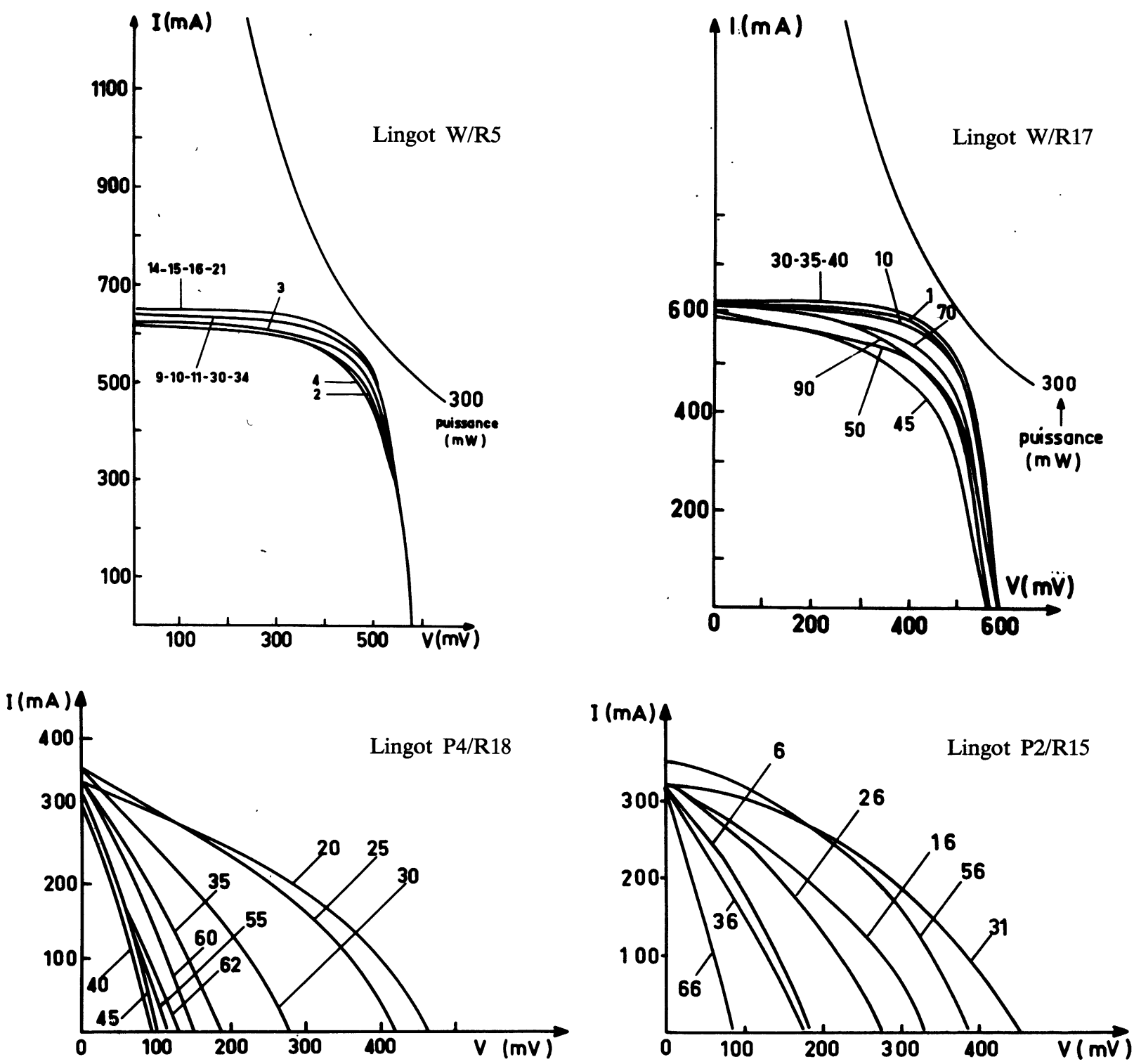

Fig. 5. - Caractéristique I(V) de cellules perpendiculaires à l'axe des lingots : W/R5 : Si-EG avec germe ; W/R17 : SiEG sans germe ; P4/R18 : Si-UMG avec germe ; P2/R15 : Si-UMG sans germe.

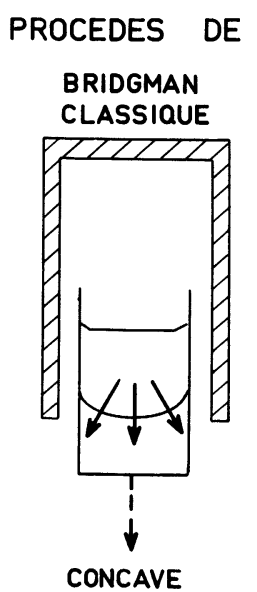

Fig. 6. - Influence de la configuration thermique sur la forme de l'interface solide liquide.

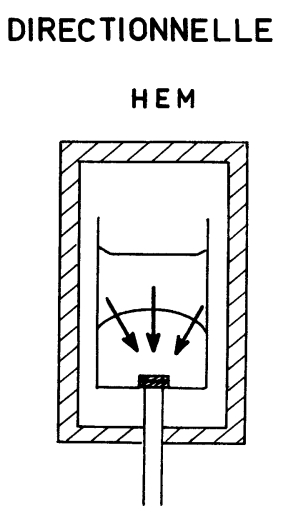

CONVEXE

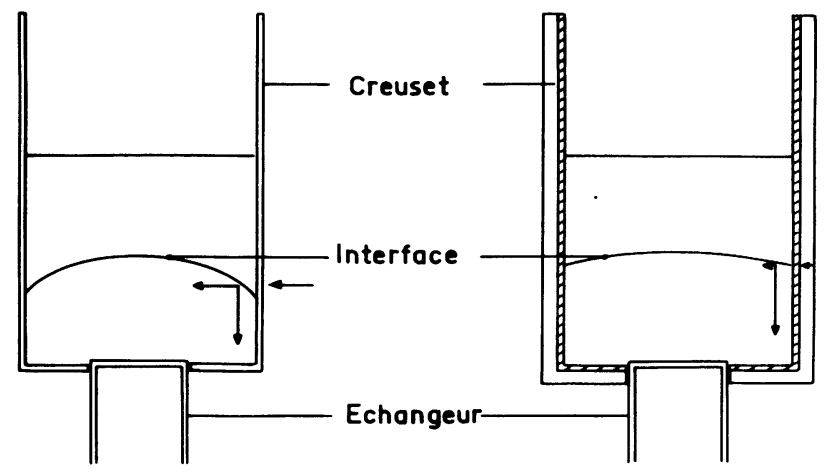

(a)

(b)

Fig. 7. - Influence du flux de chaleur radial sur la forme de l'interface : a) Creuset transparent : flux radial entrant important. b) Creuset opaque : flux radial entrant faible. 

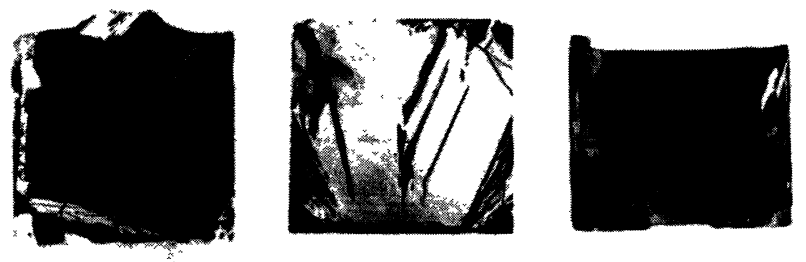

Fig. 8. - Structures cristallines effectivement obtenues pour trois lingots : a) Creuset transparent (silice fondue transparente). b) Creuset transparent, revêtement interne opaque conducteur. c) Creuset transparent, revêtement interne opaque isolant. Noter le rétrécissement de la partie centrale monocristalline de a) en c).

\section{Comportement des impuretés au cours du traite- ment.}

Nous avons voulu vérifier que le modèle dans lequel on admet que la concentration dans le liquide est homogène et celle dans le solide $\left(x_{S}\right)$ est donnée par la formule :

$$
x_{S}=x_{0} \times \operatorname{Keff} \times(1-g)^{\operatorname{Keff-1}}
$$

où :

Keff : coefficient de partage effectif, supposé constant dans la gamme des concentrations étudiées,

$x_{0}:$ concentration initiale,

$g$ : fraction solidifiée,

s'appliquait à la solidification directionnelle du silicium dans nos conditions expérimentales.

La vérification a été faite en calculant le coefficient de partage effectif du bore et de l'aluminium.

Pour le bore, les variations de concentration sont déduites des mesures de résistivité électrique dans les grains. Le coefficient effectif ainsi mesuré est de 0,81 , pour un coefficient de partage théorique à l'équilibre $K_{0}=0,8$.

Pour l'aluminium, un lingot a été volontairement enrichi de $1600 \mathrm{ppm}$ atomiques. Dans ce cas, dans la plus grande partie du lingot le modèle ne s'applique pas. Dans la partie inférieure du lingot, aux faibles concentrations, l'utilisation de cette formule conduit à $K e f f \leqslant 4 \times 10^{-3}\left(K_{0}=2 \times 10^{-3}\right)$.

Dans le cas d'autres impuretés métalliques, il est difficile de vérifier ce modèle et de mesurer des valeurs de Keff. En effet :

- A partir de Si-EG, malgré la très grande sensibilité des méthodes d'analyse par activation neutronique, un grand nombre d'impuretés ne sont pas décelées dans la plus grande partie de nos lingots. De plus, des pollutions par les parois du creuset sont visibles à la périphérie des lingots et traduisent un enrichissement pendant la fusion, ce qui modifie les valeurs des $x_{0}$ initialement sur le SiEG de départ.
- A partir de Si-UMG, le matériau de départ est souvent très hétérogène, les $x_{0}$ variant d'un facteur 10 à 100 . De plus, certaines impuretés paraissent retenues dans la partie inférieure des lingots, région à plus petits grains et où des précipités de SIC ont été mis en évidence à la microsonde électronique (tailles de quelques microns à quelques dizaines de microns). Néanmoins, il est certain que la migration des impuretés métalliques est très prononcée lors de la solidification directionnelle du silicium. L'importance de cette migration semble liée à la valeur du coefficient de partage à l'équilibre $K_{0}$. Toutefois, les coefficients réels d'élimination en différents points des lingots (rapport de la teneur en ce point à la teneur initiale) peuvent être supérieurs de plusieurs ordres de grandeur à ceux calculés à partir de la formule ci-dessus en utilisant les $K_{0}$ correspondants. Tel est par exemple le cas du nickel pour lequel nous trouvons dans nos lingots des coefficients d'élimination de l'ordre de $10^{-2}$ alors que la valeur du coefficient de partage à l'équilibre $\left(K_{0}\right)$ est de l'ordre de $10^{-4}$.

Il est donc abusif de déduire a priori la teneur en impuretés résiduelles d'un lingot de silicium recristallisé directionnellement en utilisant la formule indiquée plus haut, et à plus forte raison en multipliant simplement la teneur initiale des impuretés par la valeur de leur $K_{0}$.

\section{Possibilités de partir directement du Si-UMG.}

Il est très tentant d'essayer d'utiliser directement le silicium de qualité métallurgique solidifié directionnellement pour préparer des photopiles. Une économie substantielle serait alors réalisée en évitant l'étape de purification chimique de ce silicium.

Nos essais faits à partir de plusieurs qualités de silicium métallurgique montrent que les impuretés métalliques sont ainsi fortement éliminées de plus de $90 \%$ des lingots. Par contre, les résistivités électriques demeurent très faibles (de l'ordre de $0,04 \Omega . \mathrm{cm}$ ) et les rendements de conversion mauvais $(<3 \%$ ) (Tabl. II, Fig. 5).

Les mauvaises propriétés photoélectriques mesurées sur les lingots préparés à partir de Si-UMG peuvent s'expliquer par la seule présence de bore. Une teneur de 1 à $3 \times 10^{18}$ at. $\mathrm{cm}^{-3}$ dans le matériau de départ est tout à fait rédhibitoire. Même en partant d'un silicium métallurgique très purifié en impuretés par une fusion plasma [23], les propriétés électriques demeurent très mauvaises car le bore n'avait pas été suffisamment éliminé du silicium dans cet essai. Plusieurs laboratoires étrangers ont abouti à des conditions similaires et font des efforts importants pour diminuer la teneur en bore de leur SiUMG, soit en sélectionnant les matériaux de départ $[11,24]$, soit en les purifiant [25].

Il est possible que ces travaux aboutissent à 
Tableau II. - Récapitulation des résultats obtenus sur diverses fournitures de silicium.

\begin{tabular}{|c|c|c|c|c|c|c|}
\hline & \multicolumn{2}{|c|}{ EG-SI } & \multicolumn{2}{|c|}{ UMG-SI } & \multicolumn{2}{|c|}{ UMG-SI + PLASMA } \\
\hline & avant & après $\left({ }^{1}\right)$ & avant & après $\left({ }^{1}\right)$ & avant & après $\left({ }^{2}\right)$ \\
\hline $\begin{array}{l}\mathrm{Co} \\
\mathrm{Cr} \\
\mathrm{Cu} \\
\mathrm{Fe} \\
\mathrm{Mn} \\
\mathrm{Mo} \\
\mathrm{Na} \\
\mathrm{Ni} \\
\mathrm{Ti} \\
\mathrm{W} \\
\mathrm{Zn}\end{array}$ & $\begin{array}{r}4 \times 10^{-5} \\
\quad 10^{-4} \\
<4 \times 10^{-4} \\
<1 \times 10^{-2} \\
<3 \times 10^{-4} \\
<2 \times 10^{-5} \\
<2 \times 10^{-3} \\
<7 \times 10^{-3} \\
<\times 10^{-1} \\
<4 \times 10^{-7} \\
<4 \times 10^{-4}\end{array}$ & $\begin{array}{r}5 \times 10^{-3} \\
<10^{-3} \\
2 \times 10^{-2} \\
<7 \times 10^{-2} \\
<5 \times 10^{-4} \\
<1 \times 10^{-4} \\
<8 \times 10^{-4} \\
6 \times 10^{-3} \\
<3 \times 10^{-2} \\
<3 \times 10^{-7} \\
<3 \times 10^{-3}\end{array}$ & $\begin{array}{c}10^{-2}-10^{-1} \\
10^{-1}-10 \\
10^{-1}-1 \\
1-10 \\
10^{-2}-1 \\
10^{-1} \\
1-10 \\
<7 \times 10^{-2} \\
1-10 \\
10^{-4}-10^{-2} \\
10^{-2}-1\end{array}$ & $\begin{aligned} & 9 \times 10^{-3} \\
& 2 \times 10^{-2} \\
& 1 \times 10^{-2} \\
< & 0,1 \\
< & 3 \times 10^{-2} \\
< & 4 \times 10^{-4} \\
< & 2 \times 10^{-3} \\
< & 7 \times 10^{-2} \\
< & 9 \times 10^{-1} \\
< & 3 \times 10^{-7} \\
< & 6 \times 10^{-3}\end{aligned}$ & $\begin{aligned} & 8 \times 10^{-4} \\
& 6 \times 10^{-4} \\
2 & \times 10^{-2} \\
< & 2 \times 10^{-2} \\
< & 2 \times 10^{-3} \\
< & 4 \times 10^{-2} \\
< & 1 \times 10^{-2} \\
< & 5 \times 10^{-3} \\
& - \\
& - \\
< & 2 \times 10^{-3}\end{aligned}$ & $\begin{array}{r}3 \times 10^{-3} \\
8 \times 10^{-2} \\
3 \times 10^{-2} \\
3 \times 10^{-2} \\
5 \times 10^{-4} \\
<1 \times 10^{-1} \\
3 \times 10^{-2} \\
7 \times 10^{-2} \\
- \\
- \\
<5 \times 10^{-4}\end{array}$ \\
\hline Bore & - & 1,7 & $100-300$ & 260 & - & 160 \\
\hline $\mathrm{f} \Omega . \mathrm{cm}$ & - & 1,4 & - & 0,037 & 0,1 & 0,04 \\
\hline $\begin{array}{l}\mathrm{V}_{\mathrm{oc}}(\mathrm{mV}) \\
\mathrm{I}_{\mathrm{cc}} \mathrm{mA} / \mathrm{cm}^{2} \\
\mathrm{~L}_{\mathrm{D}}(\mu \mathrm{m}) \\
\mathrm{R} \% \\
\mathrm{FF} \%\end{array}$ & & $\begin{array}{c}578 \\
25 \\
65-120 \\
9,8 \\
69\end{array}$ & & $\begin{array}{l}<500 \\
<15 \\
<3 \\
-\quad 3\end{array}$ & & $\begin{array}{c}427 \\
20,2 \\
18-25 \\
3,3 \\
38\end{array}$ \\
\hline
\end{tabular}

( ${ }^{1}$ ) Lingots : $\varnothing 100 \mathrm{~mm} ; h=80 \mathrm{~mm}$; poids $=1,46 \mathrm{~kg}$.

$\left({ }^{2}\right)$ Lingots : $\varnothing 52 \mathrm{~mm} ; h=63 \mathrm{~mm}$; poids $=0,31 \mathrm{~kg}$.

ramener la teneur en bore à des valeurs acceptables, mais le carbone, présent à des concentrations largement supérieures à sa limite de solubilité, risque de compromettre encore les possibilités d'utiliser ce matériau pour préparer directement des photopiles. En effet, les précipités de SiC sont probablement responsables de germinations parasites qui se traduisent par une structure à petits grains, de la rétention de certaines impuretés à grande affinité pour le carbone $(\mathrm{Co}, \mathrm{Cr}, \mathrm{Fe}, \mathrm{W} . .$.$) , de courts-circuits.$

La présence de gros précipités de $\mathrm{SiC}$ (de l'ordre de quelques dizaines de microns) dans le bas des lingots préparés à partir de Si-UMG a été mise en évidence par les sondes électronique et ionique. La présence de précipités plus petits, répartis dans l'ensemble de ces lingots, ne peut pas être exclue. Une étude par microscopie électronique à haute résolution est actuellement en cours.

Actuellement, il n'est donc pas possible d'utiliser les lingots à partir de Si-UMG pour fabriquer des photopiles par les techniques habituelles. Par contre, ces lingots présentent sur plus de $80 \%$ de leur masse une structure basaltique avec des grains de section de l'ordre du centimètre et ils sont largement purifiés en impuretés métalliques. Ils constituent donc un matériau de choix pour réaliser des dépôts en épitaxie [26]. Si ces derniers sont réalisés dans de bonnes conditions, il paraît possible d'atteindre et de dépasser des rendements de 11 et même $12 \%$. Un rendement de $10,3 \%$ a été atteint sur une plaque prélevée dans le lingot P2/R15 [27]. $\mathrm{Ce}$ résultat préliminaire est perfectible. Par ailleurs, il a été vérifié que l'aluminium, même lorsqu'il est présent dans les substrats jusqu'à des teneurs de $10^{18} \mathrm{at} . \mathrm{cm}^{-3}$, ne perturbe pas les propriétés de la couche en épitaxie [28].

Par rapport à la filière classique, la voie épitaxique peut être économiquement compétitive et intéressante en particulier pour les pays en voie de développement. Les capacités de production requises pour atteindre le seuil de rentabilité sont moindres et les investissements sont moins importants. Il ne faut cependant pas perdre de vue que les différences de coût sont faibles. Dans ces conditions, les prévisions, même à moyen terme, sont hasardeuses car un progrès décisif sur la purification de Si-UMG ou un abaissement un peu plus important que prévu des coûts de la voie chimique peuvent tout remettre en question. De plus, la stabilité des photopiles ainsi préparées (dépôt en présence de chlore) n'est pas aussi bien connue que celle des photopiles classiques. Néanmoins RCA (avec $\mathrm{SiH}_{2} \mathrm{Cl}_{2}$ ) [29] a effectué et 
Telefunken (avec $\mathrm{SiCl}_{4}$ ) [30] poursuit des études sur cette voie par épitaxie.

\section{Conclusion.}

La méthode de croissance que nous avons développée se prête bien à la préparation de lingots de silicium polycristallin destinés à un usage solaire. Pour la mise en œuvre de cette méthode, une attention particulière est à porter sur deux points : le choix du creuset et le contrôle de l'interface solideliquide au cours de la solidification.

Les creusets retenus pour cette étude ont l'avantage d'introduire peu de pollutions au cours de la fusion et peu de contraintes au cours du refroidissement; de plus, ils autorisent le démoulage à la température ambiante. Ils présentent l'inconvénient de ne pas être réutilisables, mais pour une production industrielle, il paraît possible de développer des creusets consommables de faible coût tout en obtenant un bon compromis entre l'adhérence du revêtement de nitrure à la paroi du creuset et l'absence de pollution. La conduite du procédé doit veiller à obtenir durant toute la solidification une interface solide-liquide plane ou légèrement convexe mais jamais concave.

Il est à noter que ces deux difficultés sont probablement les mêmes que celles rencontrées dans la mise en œuvre des autres procédés de solidification directionnelle en creuset. Les différentes méthodes utilisées actuellement ne diffèrent en fait les unes des autres que par la façon d'extraire la chaleur par le fond du creuset et éventuellement par la nature de ce creuset.
Le comportement des impuretés n'est pas seulement régi par une loi de ségrégation simple d'un alliage binaire. La présence d'autres impuretés et la conduite du procédé influencent ce comportement. En général les coefficients réels d'élimination obtenus expérimentalement sont moins favorables que ne le laisseraient supposer a priori les valeurs correspondantes des coefficients de partage à l'équilibre. Néanmoins, les impuretés métalliques du Si-UMG sont, pour la plupart, largement éliminées de la partie utile des lingots au cours de la solidification directionnelle. Les impuretés métalloïdiques : bore, carbone, oxygène..., le sont beaucoup moins. Aussi actuellement, il n'est toujours pas possible d'utiliser directement les lingots préparés à partir de Si-UMG pour réaliser des photopiles suivant les techniques classiques pour le silicium massif. Par contre, les plaques découpées dans les lingots ainsi obtenus constituant des substrats convenables pour réaliser des photopiles suivant la technique des dépôts en épitaxie. Des photopiles ayant un rendement supérieur à $12 \%$ devraient pouvoir être ainsi obtenues dans des conditions financières très compétitives avec la filière classique.

\section{Remerciements.}

Ce travail a été financé à la fois par l'AFME et le PIRSEM. Les auteurs remercient C. Dardenne, R. Delmas et N. Deschamps (Laboratoire C.N.R.S. « Pierre Süe », Saclay) pour les analyses par activation neutronique, et la Société Photowatt Ind. S.A. pour la réalisation et la caractérisation électrique de photopiles à partir des lingots.

\section{Bibliographie}

[1] Schmid, F., Viechnicki, D., J. Am. Ceram. Soc. 53 (1970) 528.

[2] Khattak, C. P., Schmid, F., Proc. 13th IEEE Photovoltaic Specialist Conf., Washington D.C., U.S.A., 1978 (IEEE, New York, 1978), p. 137.

[3] VIECHNICKI, D., SCHMID, F., J. Crystal Growth 11 (1971) 345.

[4] Fally, J., Guignot, D., Proc. 1st Intern. Photovoltaic Science and Engineering Conf., Kobe (Japan) 1984, p. 25.

[5] Revel, G., Pastol, J.-L., Lauvray, H., BesseLERE, J.-P., THERME, J., Rapport COMES 79.105 (1980).

[6] Revel, G., Pastol, J.-L., Hania, D., Nguyen DINH HUYNH, Rapport AFME 80.75.239 (1982).

[7] Revel, G., Pastol, J.-L., Hania, D., Rapport AFME 3.310.3800 (1985).

[8] Revel, G., Hania, D., Pastol, J.-L., Proc. MRS Conf. Poly-Microcrystalline Amorphous Semiconductors, Strasbourg, France, 1984 (Ed. de Physique, 1984), p. 147.
[9] Revel, G., Pastol, J.-L., Hania, D., NGuYen Dinh HuYnh, Proc. 5th E.C. Photovoltaic Solar Energy Conf., Kavouri, Greece, 1983 (D. Reidel, 1984), p. 1037.

[10] Saito, T., Shimura, A., Ichikawa, S., Proc. 15th IEEE Photovoltaic Specialist Conf., Orlando, U.S.A., 1981 (IEEE, New York, 1981), p. 576.

[11] Amick, J. A., Dismukes, J. P., Ravishankar, P. S., SChNeider, M., Blake, J., Matthei, K., Sylvain, R., Halvorsen, G., Larsen, K., SCHEI, A., Proc. 5th E.C. Photovoltaic Solar Energy Conf., Kavouri, Greece, 1983 (D. Reidel, 1984), p. 1068.

[12] Chemelli, C., Beghi, M., Fossati, S., Rota, F., Pizzini, S., Bigoni, L., Proc. 6th E.C. Photovoltaic Solar Energy Conf., London, U.K., 1985 (D. Reidel, 1984), p. 1036.

[13] Saito, T., Shimura, A., IChiKawa, S., Solar Energy Materials 9 (1983) 337.

[14] Revel, G., Progress in Crystal Growth and Characterisation 8 (1984) 167. 
[15] Revel, G., Deschamps, N., Dardenne, C., PasTOL, J.-L., HANiA, D., NGUYEN Dinh HUYNH, J. Radioanal. Nucl. Chem. Lett. 85 (1984) 137.

[16] Donon, J., Lauvray, H., Demongeot, F., Rapport AFME 11000.2300 (1981).

[17] Donon, J., Lauvray, H., David, G., Aubril, P., Heroult, J.-P., Proc. 5th E.C. Photovoltaic Solar Energy Conf., Kavouri, Greece, 1983 (D. Reidel, 1984), p. 1133.

[18] Mertens, R., Cheek, G., Pauw, P. De, Frisson, L., Proc. 5th E.C. Photovoltaic Solar Energy Conf., Kavouri, Greece, 1983 (R. Reidel, 1984), p. 976.

[19] Frisson, L., Cheek, G., Mertens, R., Van OverStraeten, R., Proc. 5th E.C. Photovoltaic Solar Energy Conf., Kavouri, Greece, 1983 (D. Reidel, 1984), p. 1002.

[20] Hania, D., Thèse de Doctorat de $3^{\mathrm{e}}$ cycle, Spécialité Energétique, Université Paris VII (1984).

[21] Beghi, M., Chemelli, C., Fossati, S., Pizzini, S., Proc. MRS Conf. Poly-Microcrystalline Amorphous Semiconductors, Strasbourg, France, 1984 (Ed. de Physique, 1984), p. 181.

[22] Lauvray, H., Lay, P., Nouet, G., Donon, J., Proc. 6th E.C. Photovoltaic Solar Energy Conf., London, U.K., 1985 (D. Reidel, 1984), p. 901.

[23] Morvan, D., Amouroux, J., Proc. MRS Conf. Poly-Microcrystalline Amorphous Semiconductors, Strasbourg, France, 1984 (Ed. de Physique 1984), p. 163.

[24] Pizzini, S., Rustioni, M., Proc. 6th E.C. Photovoltaic Solar Energy Conf., London, U.K., 1985 (D. Reidel, 1984), p. 875.
[25] Aulich, H. A., Bernewitz, L., FenCZ, H. J., Pink, H., Schulze, F. W., Proc. MRS Conf. Poly-Microcrystalline Amorphous Semiconductors, Strasbourg, France, 1984 (Ed. de Physique 1984), p. 175.

[26] Baulac, R., Revel, G., Hania, D., Pastol, J.-L., NGuyen Dinh Huynh, Rodot, M., Bourée, J.-E., Leray, C., Le QuANG Nam, Dubrous, F., Bargues, M., Proc. 5th E.C. Photovoltaic Solar Energy Conf., Kavouri, Greece, 1983 (D. Reidel, 1984), p. 1053.

[27] Caymax, M., Perraki-Goutsou, V., Pastol, J.L., Bourée, J.-E., Eckmans, M., Mertens, R., Revel, G., Rodot, M., Intern. Photovoltaic Solar Energy Conf., Beijing (Pekin), China (1986).

[28] Rodot, M., Revel, G., Bourée, J.-E., Pastol, J.L., Le QuAng NAM, Proc. ECS Symp. Materials and New Processing Technologies for Photovoltaics, New Orleans, U.S.A., 1984 (Electrochemical Society, 1985), p. 276.

Bourée, J.-E., Le Quang Nam, Barbé, M., PerRAKI-Goutsou, V., Rodot, M., Proc. 6th E.C. Photovoltaic Solar Energy Conf., London, U.K., 1985 (D. Reidel, 1984), p. 1051.

[29] D'Aiello, R. V., Robinson, P. M., Miller, E. A., R.C.A. Rev. 44 (1983) 30.

[30] Schmidt, W., Rasch, K. D., Roy, K., Proc. 6th E.C. Photovoltaic Solar Energy Conf., London, U.K., 1985 (D. Reidel, 1984), p. 906. 